\title{
Crafting Contemporary Enterprise Application User Experiences
}

\author{
Jeremy Ashley and Misha W. Vaughan \\ Oracle USA \\ 500 Oracle Parkway, MS 2op10 \\ Redwood Shores, CA 94065 \\ USA \\ jeremy.ashley@oracle.com, mvaughan@acm.org
}

\begin{abstract}
This paper outlines the field research program defined by an enterprise application user experience organization designed to collect data from globally distributed corporations. The goal of the research program was to drive the next generation user experience of enterprise applications. This paper discusses the top five lessons learned and their design solutions.
\end{abstract}

Keywords: enterprise applications, user experience, site visits, field studies.

\section{Historical Perspective}

Ten to 15 years ago, enterprise software applications were designed as siloed, transactional, front ends for databases. These applications were used by experts who received weeks of training and who conducted their work on big, expensive business machines. At the end of the day, what mattered most was the ability to complete a particular business task.

These applications were products of the development view of the time. Because not all end users were database programmers, applications were written to make working with the data a little easier. For the most part, these applications were direct expressions of the database, only with a graphical user interface rather than a command-line user interface. Each database-whether it was sales, service, marketing, payables, inventory, or receivables — had its own user interface.

Around 2000, the Internet began pushing those same applications to Web-based experiences. Developers began crafting HTML interfaces for those same applications [1]. Web-based businesses designed for consumers, such as Amazon.com, were also emerging. These Web-based businesses were changing the competitive landscape. As executives and end users alike saw how straightforward the user experience was, they placed the same expectation on these emerging applications [2].

Today, users' expectations have made another quantum leap. Now, when users go home at night, they procure on Ebay and invoice on Citibank. These experiences are straightforward and transparent. In addition, new technologies have emerged, such as Asynchronous JavaScript and XML (AJAX), which enable designers to build richer and more interactive experiences. Finally, new methods to document business processes have been developed, such as business process modeling (BPM), a method 
that enables us to document and design business best practices. The convergence of these elements means that we can deliver a user experience that enables users to concentrate on their jobs, not the software.

This paper describes a plan of user research for the field embarked upon by an enterprise application user experience organization to meet these emerging needs. Some of the challenges this plan had to address included: coordinating this at a group level, creating consistency across teams, and conducting the research globally. The paper discusses methodologies, deliverables, and the top five lessons learned and their related design solutions.

\section{User Research}

Starting in 2004, the Oracle Applications User Experience organization embarked on a substantial field study program to inform the design of the next generation of user experiences for enterprise applications. The field study program spanned multiple years (2004-2008), domains, and countries. The goal of the field research was to understand:

- The current state of end user experiences with a wide variety of enterprise applications

- What was successful about end user experiences that should be carried forward

- The pain points of existing enterprise software

- The workarounds that end users created when software did not meet their needs

\subsection{Scope}

To better understand the broad range of problems, the research was divided into several domains: human capital management, customer relationship management, financials, supply chain management, procurement, corporate performance monitoring, enabling technologies, mobile, and business intelligence. In each of these domains, companies were selected for observation based on their mix of enterprise applications on site and on their strategic relationship with our organization. This relationship proved to be necessary in order to negotiate the complex legal agreements required for each site visit. In total, approximately 1,300 hours of site visit data were collected from over 600 end users across 8 countries.

\subsection{Methodology}

Because we had such a large organization (approximately 150 people), we chose to create a series of best practice guidelines for designing, conducting, analyzing, and reporting on our field studies. This section covers the general recommendations for conducting the research.

Preparation. To prepare for the site visits, each Oracle user experience team was responsible for: identifying the target corporation profile, understanding prior research, obtaining legal access, scoping the functional areas within a domain, and defining the target user profiles. We will detail a specific example of these steps for the Financials domain. 
The lead usability engineer (UE) for a given domain, working with an internal strategy organization, first developed a corporation profile for each domain: corporation size, location, industry, geographies, and installed applications. In financials, the team identified the following criteria for the corporation profile:

- Revenue: annual revenue of one billion or more

- Industry: public sector, financial services, and communications

- Geographic area: North America, Europe, India, and China

- Installed enterprise applications: JD Edwards, Oracle E-Business Suite, and PeopleSoft

While completing the corporation profile, the lead UE also reviewed any publicly available or internally created user and market research data about the domain. This analysis of existing data enabled the UE to work with other members of Oracleparticularly in the strategy organization- to define the functional areas within a given domain and the user profiles of interest. For example, again in Financials, the team narrowed its interest to the following functional areas: expenses, general ledger, accounts payable, accounts receivable, and asset lifecycle management.

Table 1. Sample user role description: accounts payable manager

\begin{tabular}{llll}
\hline Job Titles & Details & Education & Computer Skills \\
\hline - Accounts & In charge of the whole & $\bullet$ Four-year & $\bullet$ Well-versed in \\
payable & accounts payable & college & Excel \\
manager & process: & accounting & $\bullet$ Functional user \\
- Disbursements & $\bullet$ Handling regular & degree & $\bullet$ Well-versed in \\
director or & and strategic & $\bullet$ Sometimes & reporting tools \\
manager & reporting, roll up & a CPA, & $\bullet$ Not necessarily \\
- Manager of & data, and volume & depending & an accounts \\
disbursements & reporting & on the size & payable expert \\
& - Dealing with special & of the & end user- \\
& accounting issues, & corporation & more focused \\
& such as checks and & & on reports and \\
& chart of accounts & & \\
& values & & \\
& Enforcing fiscal & & \\
& discipline & & \\
\hline
\end{tabular}

The team then developed several user profiles for each functional area. For example, for accounts payable, the team identified the following user roles: accounts payable manager, expenses supervisor, supplier invoices supervisor, payments supervisor, and processor. For each user role, the team created a detailed description of expected characteristics. For example, the above table details a description of an accounts payable manager (Table 1).

Starting Discussions with the Customer. The UE then embarked on the complex and lengthy task of contacting corporations with which our organization had a preexisting relationship and fit the target profile. Once a corporation expressed initial 
interest, the UE delivered a presentation to educate the customer about what would be involved. If the customer agreed to participate, it identified its own internal stakeholders and the UE would then present a specially created legal agreement that protected both parties, yet still allowed our organization to leverage the findings in the development of software user interfaces.

Next, the UE scheduled a follow up conference call and presentation for all of the stakeholders - in which the UE would once again describe for the customer what was involved in a site visit. During this same call, the UE would determine the following things about the corporation: additional stakeholders, the information technology environment, the relevant job titles, and the top user-experience issues. After several follow-up phone calls, the UE identified an executive sponsor and a logistical point of contact for the actual visits.

The UE would then begin recruiting the actual participants. The UE typically contacted managers who oversaw the needed individual contributors and gave the same presentation. The potential observation candidates were then screened to ensure that they met our user profiles.

For each site visit, which lasted approximately a week, the UE then created a detailed hour-by-hour agenda. Maps of each location and a contact list of individuals at the customer locations were all prepared in advance.

On Site. For each site visit, a team of three to seven individuals from our organization were deployed, including a UE. On day one, the UE presented a basic "Who we are and why we are here" message. This presentation was often attended by a wide variety of individuals from the corporation who were curious about the site-visit process. At the end of this meeting, a focus-group-style conversation was often held in order for customers to do the one thing most on their minds: vent their frustrations about enterprise software.

The Oracle team would then begin the one-on-one observations. Each observation included three basic components:

- Administering a brief questionnaire to understand whether our assumptions about demographics and job experience were correct.

- Observing the end users for 1 to 4 hours, depending on the kind of work performed, seeking to discover exceptions and unexpected findings

- Clarifying observations, asking to view artifacts, recording particpants photographically (if within scope of the legal agreement), and pursuing discoveries.

- Over the next 2 to 5 days, the following events and artifacts would emerge:

- The Oracle user experience team would observe each user profile for 1 to 4 hours.

- The Oracle user experience team would develop a detailed business process diagram and information technology map based on these observations and on the discussions with information technology managers at the corporation.

After the last day of observation, the Oracle team would present their preliminary findings on site to the users. This presentation was illuminating to the customer, and motivating to the Oracle team to begin the data analysis.

Data Analysis and Deliverables. Because site visit data is rich and produces a large set of artifacts, e.g., diagrams, interview notes, photographs, and transcripts, we chose 
to use affinity diagramming as a method to distill down all of this data to a manageable set. Affinity diagramming is an analytic technique used particularly in businesses to synthesize trends and patterns. For more information, please see [3].

After the affinity diagramming analysis, we asked each team to generate a particular set of deliverables that further refined our results. We asked each user experience team to construct:

- A "day-in-the-life" scenario of their primary user roles

- A presentation comparing the Oracle team's assumptions with the observed realities about the user roles and the work environment

- The key lessons learned about each user role: what the user roles wanted and needed from their enterprise applications

- A presentation documenting the top five features that our organization could develop as a means of improving enterprise application user experience

- A detailed presentation documenting the results of the questionnaire

- A chart illustrating the corporation's organizational structure

- Task flow diagrams for users based on the one-on-one observations

\section{Top Five Lessons Learned and the Design Solutions}

Based on our field research, the number one problem facing customers of enterprise applications today is end-user productivity. By conducting site visits and observational interviews with customers, we identified five problem areas impacting productivity and then came up with design solutions for how we could improve the user experience in these areas.

\subsection{End Users Spend Too Much Time Navigating between Applications, Which Translates into a Loss in Productivity}

When it comes to Oracle applications (e.g., E-Business Suite, PeopleSoft, JD Edwards, and Siebel), end users of these applications have been exposed to a variety of user interface paradigms. Navigating among these various interfaces takes time and results in lost productivity. In our next generation of designs, users are exposed to only one user interface paradigm: the user interface shell (Fig 1).

Heavily informed by PeopleSoft's user research and strategic design work, the user interface shell provides a single point of access to all of a user's applications, analytics, collaboration, search, and even help. Consistent placement, presentation, and behavior of common user interface elements in the user interface shell results in a cohesive user experience that is easily learned and used by a diverse range of workers, thus saving time and money spent on training and support.

The navigation area spanning the top of the user interface shell provides the tools that are available across the suite of applications: menu navigation, search, recent items, and favorites. The region on the left contains quick access to tasks, a targeted search, and reports. The large transaction area in the center is the focus of the user's work and contains all the information and actions required to accomplish his or her tasks. The region on the right contains tools (such as analytics and help) that are driven by the work being performed in the center area. 

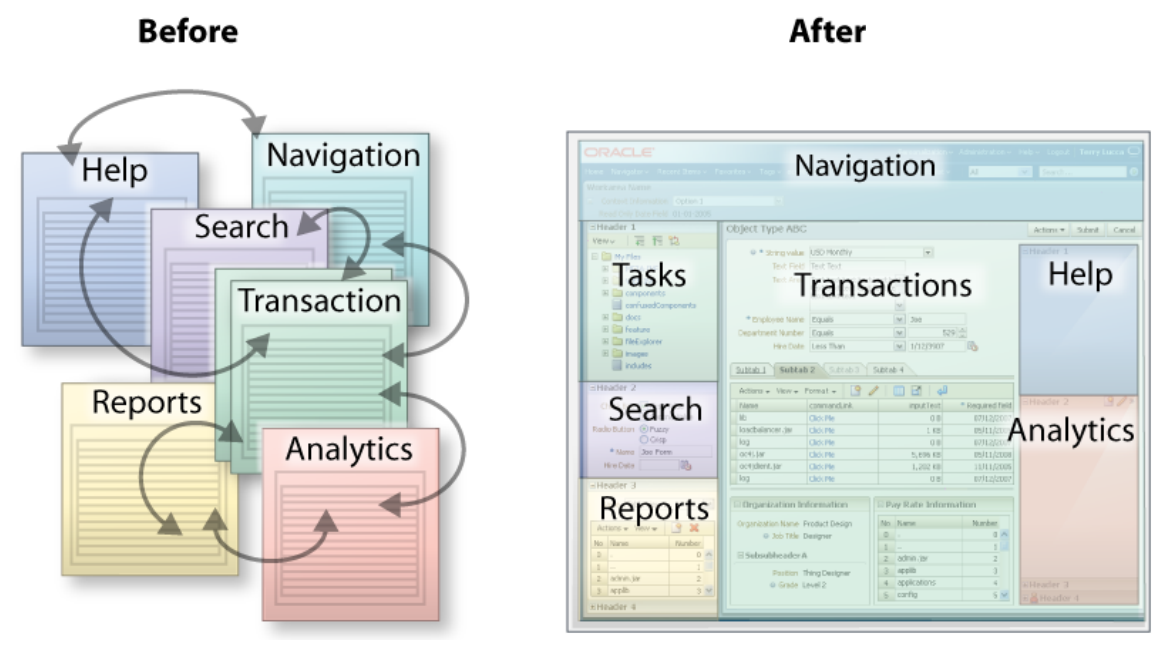

Fig. 1. How the user interface shell unifies the user experience

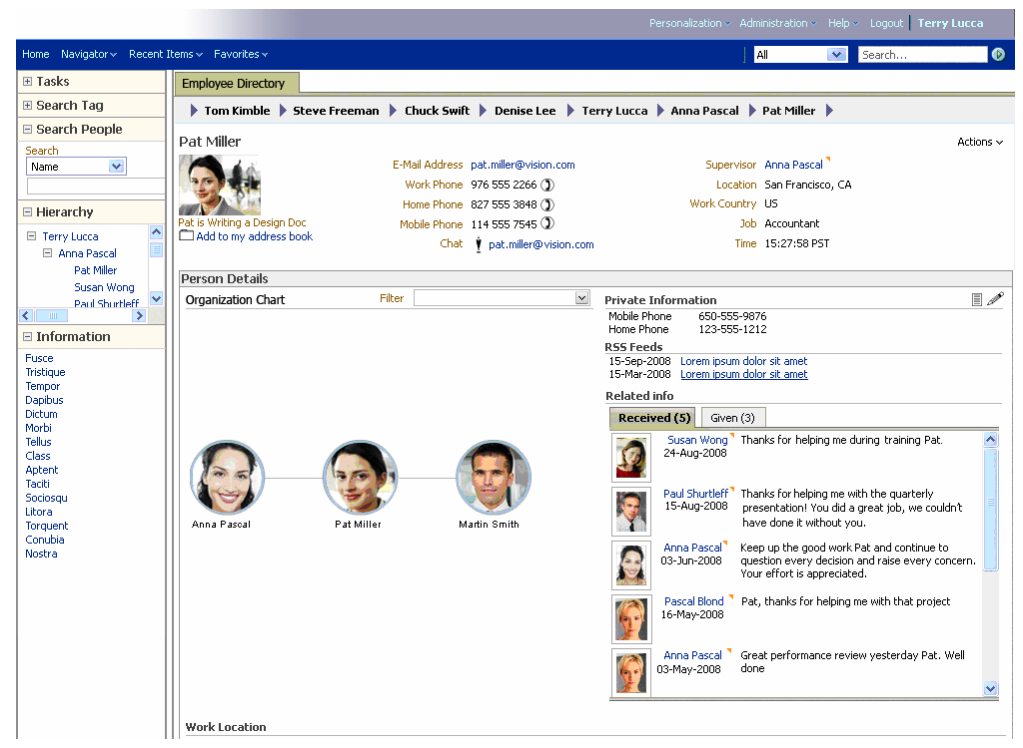

Fig. 2. Example Work Area (with right side pane collapsed) for a manager providing commonly needed information and actions together (e.g., Tasks, Search Tags, Hierarchy, Information, Employee Directory, and Person Details)

\subsection{End Users Have Clear Ideas of Business Best Practices, and They Create Work Arounds, Short Cuts, and Job Aids to Capture the Information That Is Not Reflected in Their Applications}

A deeper level of analysis of our customer data revealed that end users lack software experiences that map efficiently to business processes. End users used sticky notes, 
calendar reminders, job aids, and other documents outside the system to help them remember procedures, perform "best practices," monitor tasks, and find other information and actions in the system.

To tackle this problem, Oracle created work areas (Fig 2), where users can perform their core transactions: creating and reviewing invoices, closing books, entering time cards, managing supply chains, and so on. Work areas are context-specific assemblies of tasks, reports, analytics, and other information that help users complete core enterprise transactions. Work areas are designed from top to bottom to increase end user productivity.

\subsection{End Users' Core Transactions Happen in a Larger Context-A Context Not Reflected in the Design of Current Applications}

One of the chief complaints of end users from bioscience, manufacturing, test and measurement, high tech, public sector, and consumer goods was the need to communicate with peers on an as-needed basis, or rather the importance of supporting ad hoc collaboration. Who better for us to learn from than sales and service representatives - the masters of constant communication. By talking with sales representatives, sales managers, help desk personnel, technical support representatives in high tech, telecommunications, pharmaceuticals, manufacturing, and retail, we guided the new user experience to a core principle of keeping users in the context of the work that they are performing.

With this design goal in mind, we designed activities, notes, interactions, and contextual actions (Fig 3). These elements provide end users with the ability to work collaboratively on projects, invoices, and other such tasks and to leverage elements of social networking - all in the context of their existing transactions and using an interface that is familiar and easy to use.

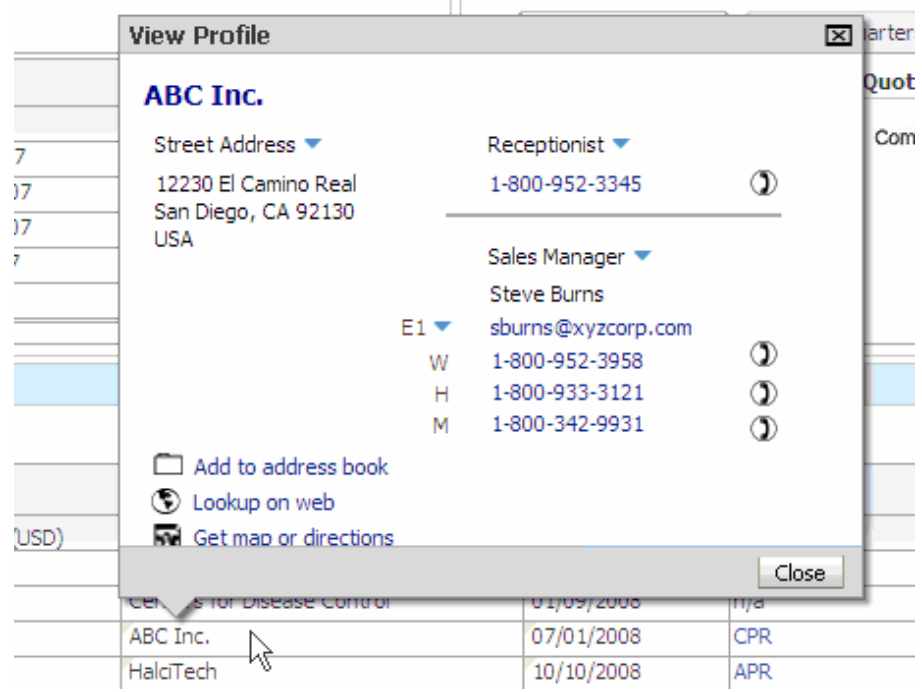

Fig. 3. Contextual actions related to a company contact 


\subsection{End Users Want to Get Work Done Faster with Rich, Interactive, Desktop- Like User Experiences}

End users often needed access to information in an enterprise application but wanted to avoid the hassle and lost time of logging in to the full enterprise application. Enter Oracle's Desktop widgets (Fig 4), beginning with search and the worklist. These desktop widgets are simple (each widget focuses on one primary task), small (unobtrusive on the desktop), and speedy (provide quick access to key information).

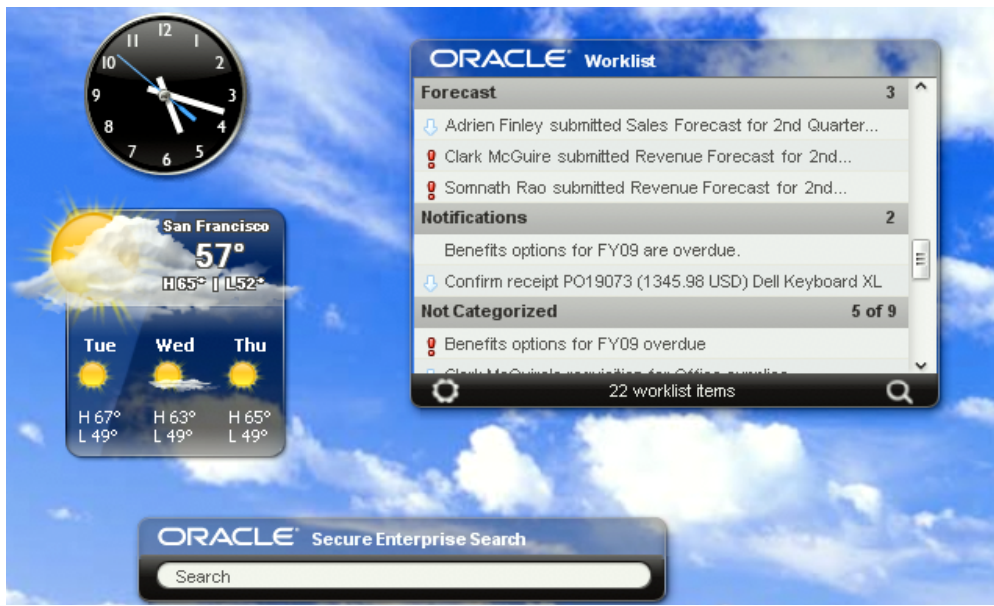

Fig. 4. Worklist widget and search widget on the desktop

\subsection{End Users Want a User Experience That Is as Easy to Use as the Internet}

In the business world, users often need to get back to a previously visited business object. For example, users would ask, "How do I go back to the last expense report that I submitted?" The Recent Items feature (Fig. 5), always presented as part of the menu in the navigation area, enables users to quickly navigate back to recently visited business objects (for example, to expense reports, purchase orders, or appraisals). Internet users take advantage of this same functionality when browsing the Web, going back to recently visited Web sites with a simple click of the mouse. Oracle's Recent Items functionality provides an instant productivity gain by enabling users to quickly and efficiently access needed information without drilling down into an application.

Along with getting back to recently used business objects, users just wanted an easy way to find information in general. "According to research firms IDC and Delphi Group, the average knowledge worker spends about a quarter of his or her day looking for information" [4]. Good search tools help them find this information (and then use it) more quickly. Users' expectations about the ease of use for search are high due to their experiences with Internet searching. At the same time, search in an enterprise context has to balance the need for security and access control. Oracle's 


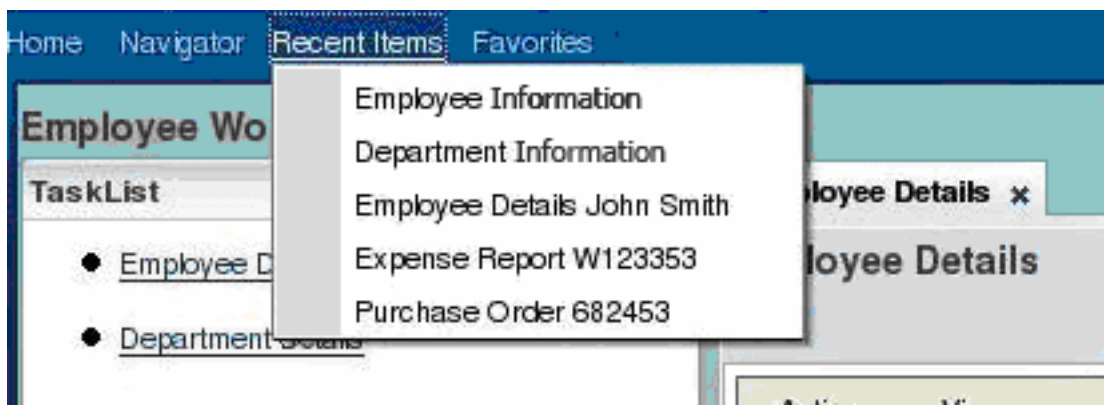

Fig. 5. Recent Items menu

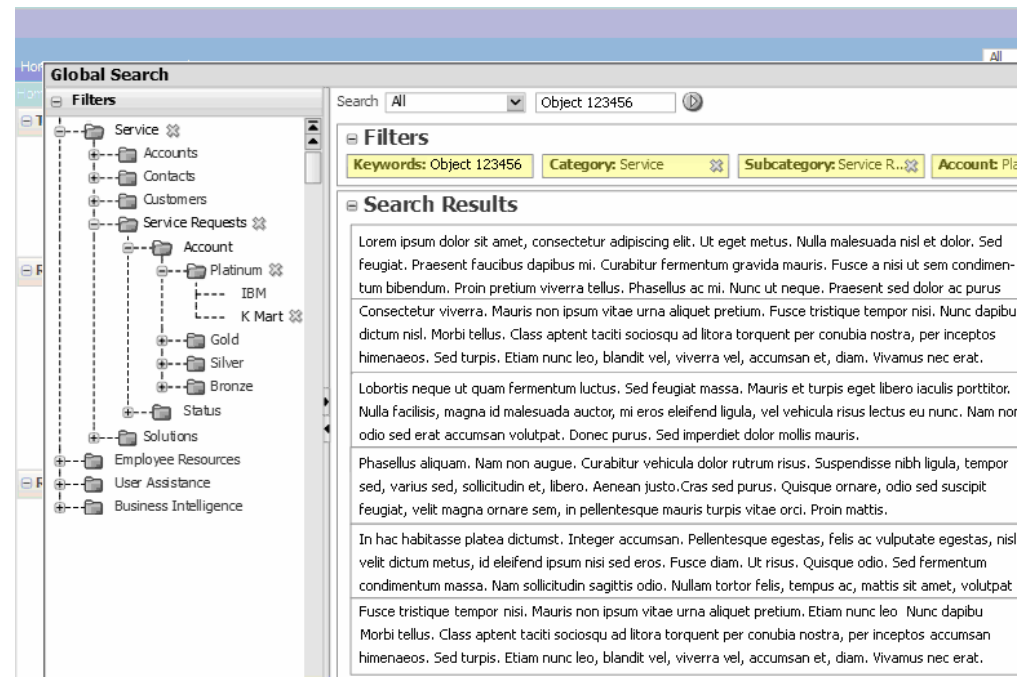

Fig. 6. Search and search results

search balances the simplicity of an Internet-like search (Fig 6) against the specificity that users of enterprise applications demand.

\section{Conclusion}

Our one key conclusion from all of our research findings is that context is important to the next generation of applications. Staying in the context of a user's work-be it sales orders, purchase contracts, or shop floor processes-radically improves productivity. This conclusion translated into a top-to-bottom design approach that provides users with the contextual information that they need to complete their tasks when they need it. 


\section{References}

1. Beier, B., Vaughan, M.W.: The Bull's-Eye: A Framework for Web Application User Interface Design Guidelines. In: Proceedings of CHI 2003: Human Factors in Computing Systems, pp. 489-496. Association for Computing Machinery, New York (2003)

2. Vaughan, M.W., Dumas, J.D.: Web-Based Programs and Applications for Business. In: Proctor, R.W., Vu, K.L. (eds.) Handbook of Human Factors in Web Design, pp. 495-513. Lawrence Erlbaum, Mahwah (2005)

3. Holtzblatt, K., Beyer, H.: Making Customer-Centered Design Work for Teams. Interactions 36, 92-103 (1993)

4. Google.: Simplicity and Enterprise Search: A New Model for Managing Your Enterprise Information (2003),

http: / /www.google.com/enterprise/pdf/

google_simplicity_enterprise_wp.pdf\#search=o22Knowledge

$\% 2$ Workers $\% 20$ Delphi 20 Group 20 Google 22 